\title{
ADAPTIVE IMPEDANCE CONTROL FOR A HYDRAULIC ROBOT WITHOUT JOINT TORQUE SENSORS BASED ON IMPROVED MINIMAL CONTROLLER SYNTHESIS ALGORITHM
}

\author{
Tomotaka ITOH*, Iwao SUZUKI**, Takashi MATSUI* \\ *Department of Mechanical Engineering, Faculty of Engineering \\ Shizuoka University \\ 3-5-1 Jouhoku, Hamamatsu, Shizuoka, 432-8561 Japan \\ (E-mail: tmtitou@ipc.shizuoka.ac.jp) \\ **ASMO CO., LTD \\ 390 Umeda, Kosai, Shizuoka, 431-0493 Japan
}

\begin{abstract}
The minimal controller synthesis (MCS) algorithm is one of the techniques of the model reference adaptive control, which is applicable for a system whose dynamic model is almost unknown. But, from our previous experimental study, we found out two problems in the practical implementation of MCS algorithm. The first is that the error convergence is prescribed by the dynamic characteristic of the reference model. The second is the nonlinearity in which the control characteristic of the system depends on the allocation of the origin of coordinates for the state variables. In this paper, the improved MCS algorithm for solving the problems is proposed. It is experimentally applied to the adaptive impedance control of a hydraulic robot without joint torque sensors and the experimental results illustrate the validity of the proposed algorithm. Although, in the impedance control, the torque control system using joint torque sensors is usually needed, our results confirms that the robustness of MCS algorithm makes it possible to implement the adaptive impedance control for hydraulic robots without joint torque sensors.
\end{abstract}

\section{KEY WORDS}

Hydraulic robot, Minimal Controller Synthesis (MCS), Adaptive Impedance Control

\section{INTRODUCTION}

The minimal controller synthesis (MCS) algorithm, introduced by D.P.Stoten et al. [1], is one of the design techniques of the model reference adaptive control (MRAC), and its extended form of MCS and the experimental investigation have been reported [2][3][4]. In the MCS, the closed-loop stability of the control system is guaranteed based on the hyperstability theorem and it is the key property that the nominal model of the plant is not needed in the controller design. Therefore, the algorithm is applicable for a system whose dynamic model is almost unknown, except for that the order of the reference model must agree with that of the plant. MCS has the possibility of applying to various robotic systems because of the advantage of easy implementation.

However, from our previous experimental study, we found out two problems in the practical implementation of MCS algorithm. The first is the error convergency problem. In the original MCS algorithm, the convergence characteristic of the error dynamics is prescribed by the dynamic characteristic of the reference model and they cannot be handled separately. Therefore, the control precision of the resultant system depends significantly on 
the choice of the reference model. The second is the nonlinearity problem. The control characteristic of the MCS-based system depends on the allocation of the origin of the coordinate system for the state variables. In the original adaptation law, the magnitudes of the signals of the state variables affect the adaptation rate of the control gain directly. Thus, the inadequate choice of the origin of the coordinates leads to a decrease of the adaptation performance around the origin. These problems may be an obstacle to the practical implementation of MCS in the engineering field.

In this paper, we'll show the outline of the original MCS algorithm and discuss the convergency and nonlinearity problems, first. Then, we propose the improved version of the MCS algorithm as a solution of these problems. Next, we design the adaptive impedance control for a hydraulic robot without joint torque sensors based on the improved MCS algorithm. In the impedance control, the torque control system with joint torque sensors is usually needed. However, in this paper, the impedance control system is designed based on the static model of the hydraulic system and we confirm that the robustness of MCS algorithm makes it possible to implement the adaptive impedance control for hydraulic robots without joint torque sensors. The proposed method is experimentally applied to a 2 -link hydraulic robot and the validity of the proposed method is illustrated.

\section{MINIMAL CONTROLLER SYNTHESIS (MCS) ALGORITHM}

The MCS algorithm[1] is one of the techniques of the model reference adaptive control and it is applicable for a system whose dynamic model is almost unknown. First, we describe the outline of the original MCS algorithm.

Generally, an $n$-D.O.F. controllable plant with order $l$ is represented as

$$
\begin{aligned}
& \dot{X}(t)=A(t) X(t)+B(t) U(t)+B_{d} d(X(t), t) \\
& X(t)=\left[x_{1}^{T} \cdots x_{l}^{T}\right]^{T}, \quad x_{i}=\left[x_{i_{1}} \cdots x_{i_{n}}\right]^{T}, \quad x_{i}=\frac{d^{i-1} x_{1}}{d t^{i-1}} \\
& A(t)=\left[\begin{array}{ccc}
0_{(l-1) n, n} & & I_{(l-1) n} \\
-A_{1}(t) & \cdots & -A_{l}(t)
\end{array}\right], \quad B(t)=\left[\begin{array}{c}
0_{(l-1) n, n} \\
b_{1}(t)
\end{array}\right], \\
& B_{d}=\left[\begin{array}{c}
0_{(l-1) n, n} \\
I_{n}
\end{array}\right]
\end{aligned}
$$

where $X(t) \in \Re^{n l}$ and $U(t) \in \Re^{n}$ represent a state variable vector and an input vector of the controlled plant respectively. $d(X(t), t) \in \Re^{n}$ is a vector which represents the effect of the external disturbance and the modeling error of the plant, etc. $A_{i}(t), b_{1}(t) \in \mathfrak{R}^{n \times n}$ are time variant coefficient matrices. Rewriting the above time-variant system with the constant matrices $A_{0}, B_{0}$ and the time-variant terms $\delta A(t), \delta B(t)$, that is
$A(t)=A_{0}+\delta A(t)$ and $B(t)=B_{0}+\delta B(t)$, we have

$\dot{X}(t)=\left(A_{0}+\delta A(t)+\delta A_{d}(t)\right) X(t)+\left(B_{0}+\delta B(t)\right) U(t)$

where the disturbance term, $B_{e} d(X(t), t)$, was assumed that it could be represented as $\delta A_{d}(t) X(t)$ with the time-variant matrix $\delta A_{d}(t)$.

Now, the reference model of the same order as the plant is defined as

$$
\begin{aligned}
& \dot{X}_{m}(t)=A_{m} X(t)+B_{m} R(t) \\
& X_{m}(t)=\left[x_{m_{1}}^{T} \cdots x_{m_{l}}^{T}\right]^{T}, x_{m_{i}}=\left[x_{m_{i_{1}}} \cdots x_{m_{i_{n}}}\right]^{T}, x_{m_{i}}=\frac{d^{i-1} x_{m_{1}}}{d t^{i-1}} \\
& A_{m}=\left[\begin{array}{ccc}
0_{(l-1) n, n} & & I_{(l-1) n} \\
-A_{m_{1}} & \cdots & -A_{m_{l}}
\end{array}\right], \quad B_{m}=\left[\begin{array}{c}
0_{(l-1) n, n} \\
b_{m_{1}}
\end{array}\right]
\end{aligned}
$$

where $X_{m}(t) \in \Re^{n l}$ is a state variable of the reference model and $R(t) \in \Re^{n l}$ is a reference input signal. $A_{m_{i}}, b_{m_{1}} \in \mathfrak{R}^{n \times n}$ represent the constant matrices describing the desired response of the reference model.

The aim of the synthesis is to design a controller so that the model following error $X_{e}(t)$ converges to zero for $t \rightarrow \infty$ and a given reference input.

$$
X_{e}(t)=X_{m}(t)-X(t)
$$

In the general model reference adaptive control based on the hyperstability design, the controller is given as

$$
U(t)=\left(K_{x}+\partial K_{x}(t)\right) X(t)+\left(K_{r}+\partial K_{r}(t)\right) R(t)
$$

where $K_{x}, K_{r}$ are constant gain matrices calculated from the nominal model of the controlled plant and $\partial K_{x}, \partial K_{r}$ are the adaptive gains for error compensation. Therefore, in the design of $K_{x}, K_{r}$, one must identify the nominal plant model. In contrast, the MCS algorithm is designed so that it guarantees the stability of the control system, even if $K_{x}, K_{r}$ are set as $K_{x}=0, K_{r}$ $=0$. Thus, it does not need the identification procedure.

$$
U(t)=\partial K_{x}(t) X(t)+\partial K_{r}(t) R(t)
$$

From Eq. (2)(3)(4)(6), the error dynamics in MCS is

$$
\begin{aligned}
\dot{X}_{e}(t)= & A_{m} X_{e}(t)+B_{e} w(t) \\
B_{e} w(t)= & {\left[\begin{array}{c}
0_{(n-1) l, l} \\
I_{n}
\end{array}\right] w(t) } \\
= & \left(A_{m}-A(t)+\partial A_{d}(t)-B(t) \partial K_{x}(t)\right) X(t) \\
& +\left(B_{m}-B(t) \partial K_{r}(t)\right) R(t)
\end{aligned}
$$

Defining the output of the error system as $y_{e}=C_{e} X_{e}$, the total error system is considered to be a Lur'e system 
(Figure 1). From the hyperstability theorem, the error dynamics is guaranteed to be asymptotically stable if the linear block $\left\{A_{m}, B_{e}, C_{e}\right\}$ is strictly positive real and the nonlinear feedback block satisfies the passivity condition:

$$
\int_{0}^{T} y_{e}(t) w(t) d \tau \geq-\gamma^{2},{ }^{\forall} T \geq 0
$$

In the MCS algorithm, using the positive definite solution $P$ of the Lyapunov equation Eq.(9) for an arbitrary positive definite matrix $Q$ and selecting $C_{e}$ in Eq.(10) make the linear block strictly positive real.

$$
\begin{aligned}
& P A_{m}+A_{m}^{T} P=-Q \\
& C_{e}=B_{e}{ }^{T} P
\end{aligned}
$$

Designing the adaptive gains $\partial K_{x}$ and $\partial K_{r}$ as

$$
\left\{\begin{array}{l}
\partial K_{x}=\alpha_{x} \int_{0}^{t} y_{e} X^{T} d \tau+\beta_{x} y_{e} X^{T} \\
\partial K_{r}=\alpha_{r} \int_{0}^{t} y_{e} R^{T} d \tau+\beta_{r} y_{e} R^{T}
\end{array}\right.
$$

the nonlinear adaptive block satisfies the passivity condition and, therefore, the error dynamics of the MCS becomes asymptotically stable, $X_{e} \rightarrow 0$.

\section{ERROR CONVERGENCY AND NONLINEARITY PROBLEMS AND IMPROVED MCS ALGORITHM}

From our previous experimental study, we found out two problems in the practical implementation of MCS algorithm. In this section, we discuss the convergency and nonlinearity problems of the original MCS and propose the improved version of the algorithm.

First, we discuss the error convergency problem. In the original MCS algorithm, the error dynamics is represented as shown in Eq.(7) and the convergence characteristic of the error dynamics is prescribed by the system matrix $A_{m}$ of the reference model. Thus, the error convergence and the dynamic characteristic of the reference model cannot be handled separately.

Now, we introduce the convergency regulation matrix, $A_{e}$, and define the modified control input as follows

$$
\begin{gathered}
U(t)=\partial K_{x}(t) X(t)+\partial K_{r}(t) Z(t) \\
Z(t)=R(t)+B_{m}^{+}\left(A_{m}-A_{e}\right) X_{m}(t)
\end{gathered}
$$

where $A_{e} \in \Re^{n l \times n l}$ is stable matrix and $B_{m}^{+}$is the pseudo-inverse matrix of $B_{m}$. From Eq. $(2)(3)(4)(12)$, the modified error dynamics is

$$
\begin{aligned}
\dot{X}_{e}(t)= & A_{e} X_{e}(t)+B_{e} w(t) \\
B_{e} w(t)= & \left(A_{e}-A(t)+\partial A_{d}(t)-B(t) \partial K_{x}(t)\right) X(t) \\
& +\left(B_{m}-B(t) \partial K_{r}(t)\right) Z(t)
\end{aligned}
$$

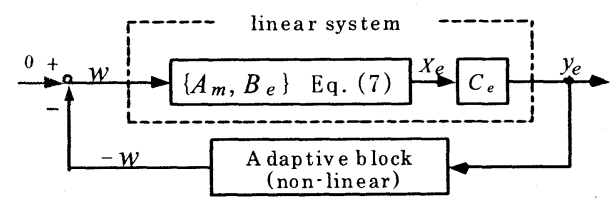

Figure 1 Block diagram of the error system

and, as a result, the error convergence can be freely prescribed by $A_{e}$. By modifying the adaptive gain

$$
\left\{\begin{array}{l}
\partial K_{x}=\alpha_{x} \int_{0}^{t} y_{e} X^{T} d \tau+\beta_{x} y_{e} X^{T} \\
\partial K_{z}=\alpha_{z} \int_{0}^{t} y_{e} Z^{T} d \tau+\beta_{z} y_{e} Z^{T}
\end{array}\right.
$$

and using the Lyapunov equation $P A_{e}+A_{e}^{T} P=-Q$, they do not disturb the hyperstability condition.

Secondly, we discuss the nonlinearity problem. In the original MCS algorithm, the adaptive gains are calculated based on the product of $y_{e}$ and $X$ or $R$ (or $Z$ ) as shown in Eq.(11)(15). Therefore, the inadequate choice of the origin of the coordinates for the state variables leads to a decrease of the adaptation performance around the origin because the magnitudes of the state variable $X$ and the reference input $R$ (or $Z$ ) affect the adaptation rate of the control gain directly.

Now, we introduce the extended gain tuning parameters by re-defining $\alpha_{x}, \beta_{x}, \alpha_{z}, \beta_{z}$ as

$$
\left\{\begin{array} { l } 
{ \alpha _ { x } = \frac { \alpha _ { x } ^ { \prime } } { \varepsilon + | X | ^ { 2 } } } \\
{ \beta _ { x } = \frac { \beta _ { x } ^ { \prime } } { \varepsilon + | X | ^ { 2 } } }
\end{array} \quad \left\{\begin{array}{l}
\alpha_{z}=\frac{\alpha_{z}^{\prime}}{\varepsilon+|Z|^{2}} \\
\beta_{z}=\frac{\beta_{z}^{\prime}}{\varepsilon+|Z|^{2}}
\end{array}\right.\right.
$$

where $\varepsilon$ is a small positive constant and $\alpha_{x}^{\prime}, \beta_{x}^{\prime}$, $\alpha_{z}^{\prime}, \beta_{z}^{\prime}$ are the extended gain tuning parameters. In Eq.(16), smaller $X$ and $Z$ increase the gain tuning parameters $\alpha$ and $\beta$ automatically. Then the decrease of the adaptation performance can be prevented.

The validity of the proposed improved MCS algorithm is confirmed in the following section of the experiments.

\section{DESIGN OF ADAPTIVE IMPEDANCE CONTROL FOR HYDRAUILIC ROBOT WITHOUT JOINT TORQUE SENSORS}

In this section, the adaptive impedance control based on the improved MCS algorithm for a hydraulic robot with no joint torque sensor is designed.

First, we describe the dynamics of the controlled plant for the explanation of the controller design, although the only knowledge, which is necessary to the MCS, is the order of the plant. In general, the dynamics of the robot manipulator is formulated as follows

$$
M(q) \ddot{q}+D(q, \dot{q})=\tau_{a}+J^{T}(q) f_{e x t}
$$


where $M$ is an inertia matrix and $D$ is a vector including the nonlinear centrifugal, Corioli's and gravity terms. $q$ is a joint angle vector and $\tau_{a}$ is a joint torque vector. $J$ is a Jacobian matrix. $f_{e x t}$ is the force vector applied to the end effector by the environment.

In the reference flame of the robot's end effector, the dynamics is re-written as

$$
\begin{aligned}
& \bar{M}(q) \ddot{x}+\bar{D}(q, \dot{q})=f_{a}+f_{e x t} \\
& \tau_{a}=J^{T} f_{a}, \quad \bar{M}(q)=J^{-T} M J^{-T} \\
& \bar{D}(q, \dot{q})=J^{-T}\left(D \dot{q}^{-1} J^{-1}-M J^{-T} \dot{J}\right) J^{-1}
\end{aligned}
$$

where $x$ is a position vector of the end effector. Now, we define the reference impedance model as

$$
M_{d} \ddot{x}_{m}+C_{d}\left(\dot{x}_{m}-\dot{r}\right)+K_{d}\left(x_{m}-r\right)=f_{\text {ext }}
$$

where $x_{m}$ is the position vector and $r$ is the reference trajectory of the end effector. $M_{d}, C_{d}, K_{d}$ are desired impedance parameter matrices.

In the state space, Eq.(18) and (19) are

$$
\begin{aligned}
& \dot{X}=A X+B U \text { and } \dot{X}_{m}=A_{m}+B_{m} R \\
& X=\left[\begin{array}{ll}
x^{T} & \dot{x}^{T}
\end{array}\right], X_{m}=\left[\begin{array}{ll}
x_{m}^{T} & \dot{x}_{m}^{T}
\end{array}\right] \\
& U=f_{a}+f_{\text {ext }}, R=K_{d} r+C_{d} \dot{r}+f_{\text {ext }} \\
& A=\left[\begin{array}{cc}
0 & I \\
0 & -\bar{M}^{-1} \bar{D}
\end{array}\right], B=\left[\begin{array}{c}
0 \\
\bar{M}^{-1}
\end{array}\right] \\
& A_{m}=\left[\begin{array}{cc}
0 & I \\
-M_{d}^{-1} K_{d} & -M_{d}^{-1} C_{d}
\end{array}\right], B_{m}=\left[\begin{array}{c}
0 \\
M_{d}^{-1}
\end{array}\right]
\end{aligned}
$$

By using the control input in Eq.(12)(15)(16) and constructing the control system as shown in Figure 2, the error dynamics become asymptotically stable and the desired impedance can be attained.

In a general impedance control, joint torque control with torque sensors is usually needed. However, in this paper, we realize the impedance control for a hydraulic robot without joint torque sensors, based on the static model of the hydraulic system and the robustness of MCS.

The characteristics of the hydraulic system in Figure 3 are described as follows

$$
\begin{aligned}
& i=K_{A} e \quad, \quad Q_{L}=\operatorname{sgn}\left(P_{s} \pm P_{L}\right) C_{d} i \sqrt{\left|P_{s} \pm P_{L}\right|} \\
& Q_{L}=D_{v} \dot{q}+K_{e} P_{L} \quad, \quad \tau=D_{v} P_{L} \\
& e \quad \text { :Input voltage } \quad i \text { :Input current } \\
& K_{A} \text { :Servo amp. gain } Q_{L} \text { :Load flow rate } \\
& P_{s} \text { :Supply pressure } P_{L} \text { :Load pressure } \\
& C_{d}^{s} \text { :Flow coefficient } D_{v} \text { :Displacement } \\
& K_{e} \text { :Leakage coefficient }
\end{aligned}
$$

From the above equations and the desired joint torque $\tau_{\text {desired }}=J^{T} U$, the inversed static model of the hydraulic system from $U$ to $e$ is formulated as

$$
e=\frac{D_{v} \dot{q}+K_{e}\left(J^{T} U / D_{v}\right)}{K_{A} \xi \operatorname{sgn}\left(P_{s} \pm\left(J^{T} U / D_{v}\right)\right) C_{d} \sqrt{\left|P_{s} \pm\left(J^{T} U / D_{v}\right)\right|}}
$$

In the experiment, this equation is used for the calculation of the input to the servo amplifier.

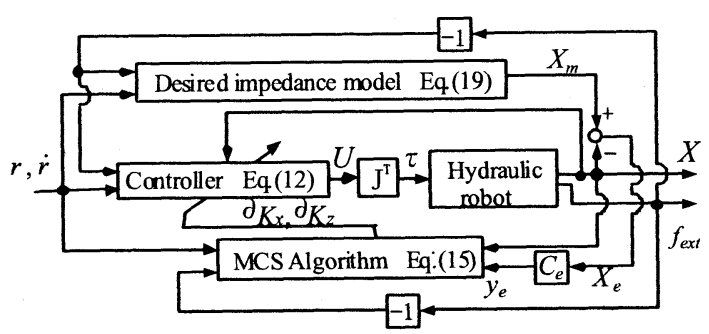

Figure 2 Adaptive impedance control system

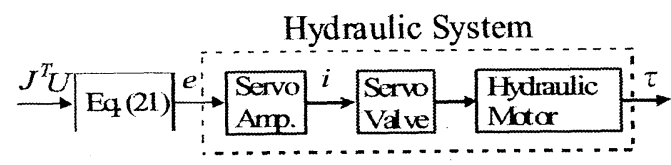

Figure 3 Block diagram of Hydraulic system

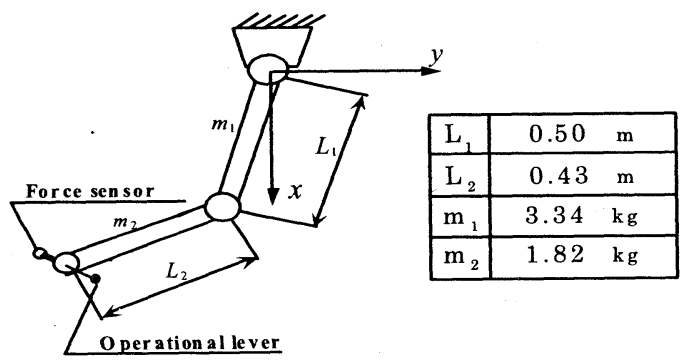

Figure 4 Experimental 2-link hydraulic robot

Table 1 Hydraulic actuators and servo valves

\begin{tabular}{|c|c|c|c|}
\hline & & Joint 1 & Joint 2 \\
\hline \multirow{3}{*}{ Actuator } & Type & \multicolumn{2}{|c|}{ Rotary actuator } \\
\cline { 2 - 4 } & Displacement & $4.55 \mathrm{~cm}^{3} / \mathrm{rad}$ & $1.79 \mathrm{~cm}^{3} / \mathrm{rad}$ \\
\cline { 2 - 4 } & Rotary angle & $\pi \mathrm{rad}$ & $\pi \mathrm{rad}$ \\
\hline \multirow{3}{*}{$\begin{array}{c}\text { Servo } \\
\text { Valve }\end{array}$} & Maker & \multicolumn{2}{|c|}{ Tokyo Prec. Instr. Co.,LTD. } \\
\cline { 2 - 4 } & Model & $401 \mathrm{~F}-114$ & $401 \mathrm{~F}-114$ \\
\cline { 2 - 4 } & Rated flow rate & $4.5 \mathrm{l} / \mathrm{min}(14 \mathrm{MPa})$ & $3 \mathrm{l} / \mathrm{min}(14 \mathrm{MPa})$ \\
\cline { 2 - 4 } & Freq. characteristic & $100 \mathrm{~Hz}(-3 \mathrm{~dB})$ & $100 \mathrm{~Hz}(-3 \mathrm{~dB})$ \\
\hline
\end{tabular}

\section{EXPERIMENTS}

The proposed adaptive impedance control algorithm was experimentally applied to a 2-link hydraulic robot with no joint torque sensor. Figure 4 shows the experimental robot manipulator. It had two degrees of freedom and each joint was directly driven by a hydraulic rotary actuator and a servo valve (Table 1). The supply pressure was $10 \mathrm{MPa}$. For the force input, an operational lever and two force sensors were attached to the end effector and 2-axis operational force $f_{h}$ and 2 axis environmental force $f_{e}$ were measured and used as the external force $f_{e x t}=f_{h}+f_{e}$. Joint rotational angles were measured by 
potentiometers and also used for the adaptive impedance control. The manipulator was controlled by a digital signal processor and its sampling rate was $1 \mathrm{~ms}$. In this paper, to confirm the validity of the proposed method, four types of experiments were executed.

[EXPERIMENT 1] Validity of convergency regulation matrix The aim of this experiment is to confirm that the proposed convergency regulation matrix $A_{e}$ realizes the free re-assignment of eigenvalues of the error dynamics. In the experiment, the reference impedance model was set as $M_{d}=\operatorname{diag}(5,5) \mathrm{kg}, C_{d}=$ $\operatorname{diag}(20,20) \mathrm{Ns} / \mathrm{m}, K_{d}=\operatorname{diag}(0,0) \mathrm{N} / \mathrm{m}$ and the adaptive impedance control algorithm was applied to the hydraulic manipulator. The positive definite matrix, $Q$, was set as follows in all experiments of this section and the gain tuning parameters were chosen as $\alpha_{x}=400, \alpha_{z}=60, \beta_{x}=2, \beta_{z}=0.2$.

$$
Q=\left[\begin{array}{cc}
10 I_{2 \times 2} & 0_{2 \times 2} \\
0_{2 \times 2} & I_{2 \times 2}
\end{array}\right]
$$

In the experiment, the response of the error dynamics was investigated under three different conditions of the eigenvalues of $A_{e}$. It should be noticed that the original MCS forces four eigenvalues to be -5 , which correspond to the eigenvalues of the reference impedance model. Figure 5 shows the tracking error responses against a step-like change of the reference position in the impedance model from the initial position $r_{0}=$ $(0.72,-027)$ to the final position $r_{f}=(0.57,-0.12)$. The dotted, broken and solid lines indicate the error responses obtained by setting four eigenvalues of $A_{e}$ under the three different conditions equally to $-3,-5,-8$ respectively. The results illustrate that the tracking error response or the error convergence can be regulated freely by introducing the regulation matrix $A_{e}$ in spite of the eigenvalues of the reference model.
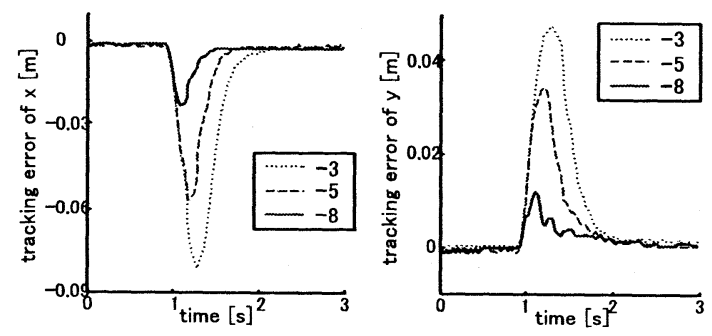

Figure 5 Tracking error on each axis

[EXPERIMENT 2] Validity of extended gain tuning parameters This experiment aims to confirm that the proposed extended gain tuning parameters can improve the nonlinearity that is derived from the origin allocation of the coordinate system of the state variables. The effects of the difference of the coordinate origin of the end effector position on the control performance were investigated in following two experiments (A) and (B), in which the control parameters were set as the same values as those used in the previous experiment. In the experiment (A), the origin of the end effector position was set as shown in Figure 4 and the tracking error response against the change of the equilibrium point from the initial position $r_{0}=(0.61,-0.37)$ to the final position $r_{f}=(0.71,-0.47)$ was obtained. In the experiment (B), we chose the origin so that it was the same position as the initial position of the experiment (A) and that the operating area was near to the origin. Therefore, the tracking target was $r_{0}=(0.0,0.0)$ and $r_{f}=(0.1,-0.1)$. In the experiments (A) and (B), all eigenvalues of $A_{e}$ was -8 and $\alpha_{x}=400, \alpha_{z}=$ $60, \beta_{x}=2, \beta_{z}=0.2$. Note that the spatial motions of the tracking target of these experiments were exactly the same. Figure 6 and 7 show the results. We can see the deterioration of the tracking performance in case that the state variables were near to the origin (Figure 7). Therefore, in the experiment (B-modified), the proposed extended parameters for gain tuning in Eq.(16) were used. (They were set as $\alpha_{x}^{\prime}=200, \alpha_{z}^{\prime}=180, \beta_{x}^{\prime}=60$, $\beta_{z}^{\prime}=40, \varepsilon=0.001$.) Figure 8 shows that the tracking performance in the area near to the origin is improved greatly and the proposed method is valid.
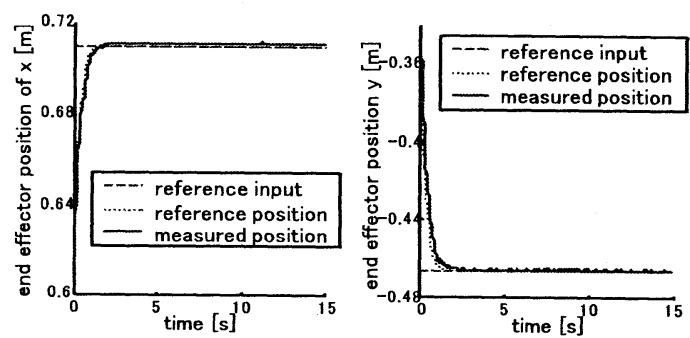

Figure 6 Experiment $(\mathrm{A})$
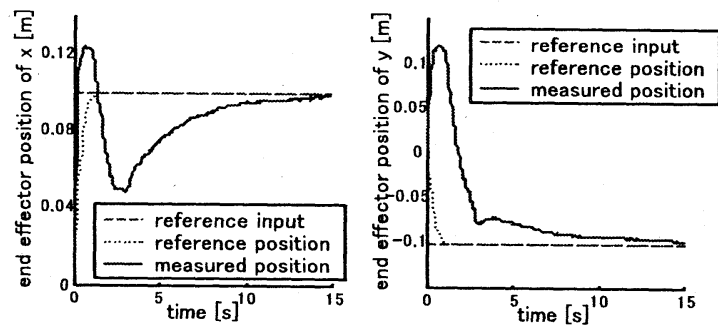

Figure 7 Experiment (B)
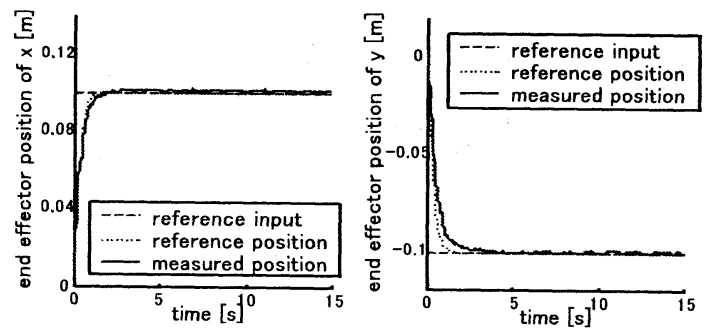

Figure 8 Experiment (B-modified) 
[EXPERIMENT 3] Impedance control in free space

The aim of this experiment is to confirm the realization of the desired impedance motion under the condition of non-constrained space $\left(f_{e}=0\right)$. In the experiment, a human operator manipulated the impedance-controlled hydraulic robot freely with the operational lever. Then, the resultant trajectory of the end effector was measured and compared to the desired trajectory, which was generated by the reference model of the impedance controller. The reference impedance model was set up as $M_{d}=\operatorname{diag}(5,5) \mathrm{kg}, C_{d}=\operatorname{diag}(20,20) \mathrm{Ns} / \mathrm{m}, K_{d}=$ $\operatorname{diag}(0,0) N / m$.

The result is shown in Figure 9. It should be noticed that the actual trajectory of the end effector (solid line) corresponds to the reference trajectory of the impedance model (dotted line) and the desired impedance was realized accurately without using joint torque sensors, because of the robustness of the adaptive MCS impedance controller.

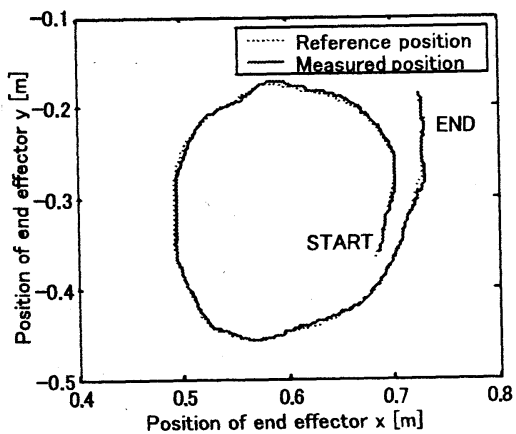

Figure 9 Trajectory in non-constrained space

[EXPERIMENT 4] Impedance control in constrained space Finally, we investigate the force response and confirm the realization of the desired compliance in the constrained space. The experimental setup is shown in Figure 10. The desired impedance of the robot was set as $M_{d}=\operatorname{diag}(10,10) \mathrm{kg}, C_{d}=\operatorname{diag}(200,200) \mathrm{Ns} / \mathrm{m}, K_{d}=$ $\operatorname{diag}(200,200) \mathrm{N} / \mathrm{m}$ and the reference trajectory of the equilibrium point of the impedance was given as shown in Figure 10. In the experiment, the robot was constrained by the flat wall and forced to follow its surface compliantly. Figure 11 shows the result. The dotted line indicates the ideal force response calculated by using the stiffness of the reference model and the solid line is the actual contact force response measured by the force sensor. Both responses are exactly the same and one can understand the proposed method is valid.

\section{CONCLUSIONS}

In this paper, the error convergence and the nonlinearity problems of the original MCS algorithm were discussed

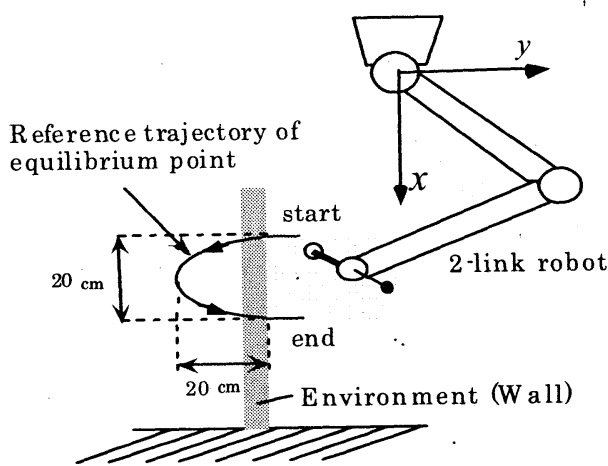

Figure 10 Experimental setup

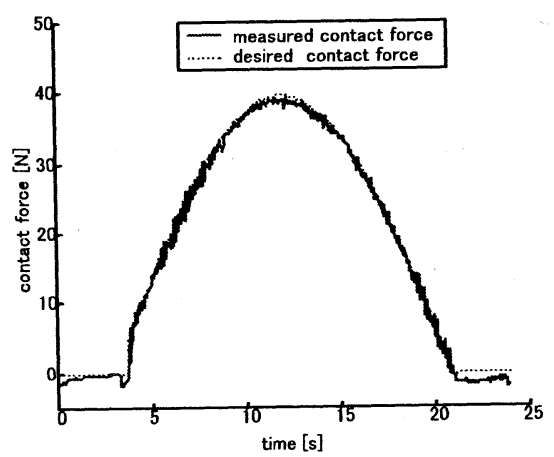

Figure 11 Contact force

and, by introducing the convergency regulation matrix $A_{e}$ and the extended gain tuning parameters, the improved version of the MCS algorithm was proposed. It was experimentally applied to the adaptive impedance control of a hydraulic robot without joint torque sensors and the experimental results illustrate the validity of the proposed method. Although, in the impedance control, the torque control with joint torque sensors is usually needed, we confirmed that the robustness of MCS algorithm made it possible to implement the adaptive impedance control for hydraulic robots without joint torque sensors.

\section{REFERENCES}

1. D.P.Stoten and H.Benchoubane, Empirical studies of an MRAC algorithm with minimal controller synthesis, Int.J. Control, 1990, 51-4, pp.823-849

2. D.P.Stoten and H.Benchoubane, Robustness of a minimal controller synthesis algorithm, Int.J. Control, 1990, 51-4, pp.851-861

3. D.P.Stoten and H.Benchoubane, The extended minimal control synthesis algorithm, Int.J. Control, 1992, 56-5, pp.1139-1165

4. D.P.Stoten and H.Benchoubane, Robustness of a minimal controller synthesis algorithm with minimal phase dynamics of unknown order but known relative degree, Int.J. Control, 1998, 71-1, pp.1-17 